Vortex-antivortex dynamics in mesoscopic symmetric and asymmetric superconducting loops with an applied ac current

This content has been downloaded from IOPscience. Please scroll down to see the full text. 2014 EPL 10857001

(http://iopscience.iop.org/0295-5075/108/5/57001)

View the table of contents for this issue, or go to the journal homepage for more

Download details:

IP Address: 146.175.11.111

This content was downloaded on 27/07/2015 at 11:04

Please note that terms and conditions apply. 


\title{
Vortex-antivortex dynamics in mesoscopic symmetric and asymmetric superconducting loops with an applied ac current
}

\author{
Guo-Qiao Zha ${ }^{1,2}$, F. M. Peeters ${ }^{2}$ and Shi-Ping Zhou ${ }^{1}$ \\ 1 Department of Physics, Shanghai University - Shanghai 200444, China \\ 2 Departement Fysica, Universiteit Antwerpen - Groenenborgerlaan 171, B-2020 Antwerpen, Belgium
}

received 16 September 2014; accepted in final form 10 November 2014

published online 28 November 2014

PACS 74.20.De - Phenomenological theories (two-fluid, Ginzburg-Landau, etc.)

PACS 74.78.Na-Mesoscopic and nanoscale systems

\begin{abstract}
In the framework of the time-dependent Ginzburg-Landau formalism, we study the dynamics of vortex-antivortex ( $\mathrm{V}-\mathrm{Av})$ pairs in mesoscopic symmetric and asymmetric superconducting loops under an applied ac current. In contrast to the case of a constant biasing dc current, the process of the $\mathrm{V}$-Av collision and annihilation is strongly affected by the time-periodic ac signal. As the direction of the applied ac current is reversed, the existed V-Av pair moves backward and then collides with a new created $\mathrm{Av}-\mathrm{V}$ pair in a symmetric loop. In the presence of an appropriate external magnetic field, a novel sinusoidal-like oscillatory mode of the magnetization curve is observed, and the periodic dynamical process of the $\mathrm{V}-\mathrm{Av}$ annihilation occurs in both branches of the sample. Moreover, for the asymmetric sample with an off-centered hole the creation point of the $\mathrm{V}-\mathrm{Av}$ pair shifts away from the center of the sample, and the creation and annihilation dynamics of $\mathrm{V}-\mathrm{Av}$ pairs turns out to be very different from the symmetric case.
\end{abstract}

Copyright (c) EPLA, 2014

Introduction. - The recent progress of modern nanofabrication techniques resulted in an increase of interests in mesoscopic superconducting samples $[1,2]$. The behavior of such superconductors is strongly influenced by the sample shape and size which, in turn, leads to new superconducting states [3-6]. A novel vortex-antivortex $(\mathrm{V}-\mathrm{Av})$ state has been predicted to exist in mesoscopic superconducting squares and triangles $[4,7,8]$. An antivortex has currents rotating in the opposite direction of a usual vortex. Normally, it is not thermodynamically stable and would annihilate with a nearby vortex. However, in the mesoscopic case the $\mathrm{V}$-Av pair may be nucleated as a consequence of the symmetry of the sample, which looks like a $\mathrm{V}$-Av molecule. The inclusion of holes into the sample can lead to enhanced properties of V-Av molecule [9]. Meanwhile, a second-generation $\mathrm{V}-\mathrm{Av}$ molecule can exist in the strategically perforated superconducting samples, which is of completely different origin from the symmetry-induced $\mathrm{V}$-Av state [10]. Also, the creation of the V-Av pair can be realized by some other ways, such as constructing the hybrid superconductor-ferromagnet structures and introducing a magnetic dipole into the system [11-16]. Moreover, the stabilized V-Av state has been demonstrated in twoband superconducting systems due to the possible coupling interactions between two bands $[17,18]$.
More recently, the dynamical behavior of the V-Av state has drawn much attention [19-24]. Sardella et al. [19] have presented the dynamics of the annihilation of a V-Av pair activated magnetically during the run of a simulated hysteresis loop on a square mesoscopic superconducting cylinder with an antidot inserted at its center. Particularly, an applied bias current can break the symmetry imposed by the sample geometry and significantly influence the vortex evolution [25]. Berdiyorov et al. [22-24] have studied the dynamics of kinematic $\mathrm{V}-\mathrm{Av}$ lines for a thin superconducting strip and a submicron superconducting loop under applied dc current, and found that the creation/annihilation point of $\mathrm{V}-\mathrm{Av}$ pairs can be manipulated by applied magnetic field and current. However, most studies done on V-Av dynamics up-to-date concern the effect of constant dc current. When the mesoscopic system is subjected to a time-periodic ac signal, new physical phenomena are expected to emerge due to the influence of ac forcing. It has been predicted that the ac component of the bias current can result in considerable modulation of the nucleation and annihilation of fluxon-antifluxon pairs in artificial stacks of SNS Josephson junctions [26,27]. Moreover, for asymmetric superconducting loops with an off-centered hole, the creation point of $\mathrm{V}$-Av pairs may shift away in comparison with the symmetric case [23]. 
Therefore, one may expect that interesting phenomena related to $\mathrm{V}-\mathrm{Av}$ dynamics in asymmetric mesoscopic loops may be present.

In the present work, we systematically investigate the V-Av dynamics in mesoscopic symmetric and asymmetric superconducting loops using the time-dependent Ginzburg-Landau (TDGL) formalism. Our numerical analysis mainly concerns the creation and annihilation of $\mathrm{V}-\mathrm{Av}$ pairs in the presence of an applied time-periodic ac current. The effect of an external magnetic field on the (anti)vortex velocity and the lifetime of $\mathrm{V}-\mathrm{Av}$ pairs is demonstrated. We also discuss the dynamical properties of the asymmetric sample with an off-centered hole. We expect our predictions may provide useful guidance for future experiments.

The model and formulas. - We consider a mesoscopic superconducting loop in the presence of an applied bias current $I$ as schematically shown in fig. 1. For an asymmetric system, $d_{l}$ and $d_{r}$ denote the off-centered displacement of the hole to the left and right side of the sample along the $y$-direction, respectively. We assume invariance of the system along the $z$-direction, which reduces the problem to two dimensions. To explore the dynamical properties of the superconducting condensate in this system, we start with the dimensionless gauge-invariant TDGL equations (eqs. (1)-(3)) [28]:

$$
\begin{aligned}
& \left(\frac{\partial}{\partial t}+i \kappa \phi\right) \psi=-\left(\frac{\nabla}{\kappa}-i \mathbf{A}\right)^{2} \psi+\psi-|\psi|^{2} \psi, \\
& \sigma\left(\frac{\partial \mathbf{A}}{\partial t}+\nabla \phi\right)=-\nabla \times \nabla \times \mathbf{A}+\mathbf{j}_{s}, \\
& \mathbf{j}_{s}=\frac{1}{2 i \kappa}\left(\psi^{*} \nabla \psi-\psi \nabla \psi^{*}\right)-|\psi|^{2} \mathbf{A},
\end{aligned}
$$

where the distance is measured in units of London penetration depth $\lambda=\left(m_{s} c^{2} / 4 \pi\left|\psi_{0}\right|^{2} e_{s}^{2}\right)^{1 / 2}$, the time $t$ in units of $\tau=\xi^{2} / D\left(\xi=\left(\hbar^{2} / 2 m_{s}|\alpha|\right)^{1 / 2}\right.$ is the coherence length and $D$ is the diffusion coefficient), the magnetic field $H$ in units of $H_{0}=\sqrt{2} H_{c}\left(H_{c}=\left(4 \pi|\alpha|\left|\psi_{0}\right|^{2}\right)^{1 / 2}\right.$ is the thermodynamic critical field strength), the vector potential $\mathbf{A}$ in units of $A_{0}=\sqrt{2} H_{c} \lambda$, the potential $\phi$ in units of $(\xi / \tau) A_{0}$, and the current density $\mathbf{j}$ in units of $j_{0}=c H_{c} / 2 \sqrt{2} \pi \lambda$. $\sigma$ is the electric conductivity and $\left|\psi_{0}\right|$ is the magnitude of the complex order parameter $\psi$ that minimizes the free energy in the absence of a field. The GL parameter $\kappa=\lambda / \xi$.

The TDGL equations can be solved by a relaxation method [29]:

$$
\begin{aligned}
\left(\frac{\partial}{\partial t}+i \kappa \phi\right) \psi & =-\frac{\partial G}{\partial \psi^{*}}, \\
\sigma\left(\frac{\partial A}{\partial t}+\nabla \phi\right) & =-\frac{1}{2} \frac{\partial G}{\partial A} \\
\sigma\left(\frac{\partial B}{\partial t}+\nabla \phi\right) & =-\frac{1}{2} \frac{\partial G}{\partial B}
\end{aligned}
$$

where $G$ is the free-energy functional, and $A$ and $B$ are the components of the vector potential $\mathbf{A}$ in the $x-y$

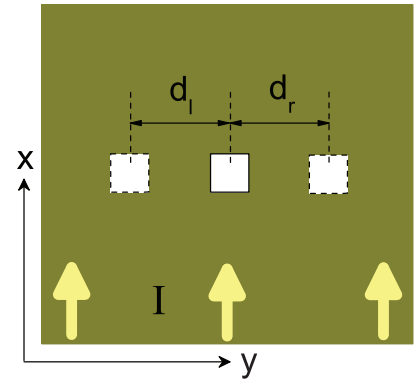

Fig. 1: (Colour on-line) Schematic view of the studied sample. $d_{l}$ and $d_{r}$ denote the displacement of the hole from the center to the left and right side of the sample perpendicular to the direction of the applied current $I$, respectively.

plane. The boundary conditions for both inner and outer boundaries are chosen as $(\nabla \times \mathbf{A}) \times \mathbf{n}=\mathbf{H} \times \mathbf{n}$ and $(\nabla / \kappa-i \mathbf{A}) \psi \cdot \mathbf{n}=-\frac{\psi}{b}$, where $\mathbf{n}$ is the unit vector normal to the sample surface and $b$ is the surface extrapolation length which is the effective penetration depth of the order parameter into the surrounding medium [30,31].

To discretize the TDGL equations and the boundary conditions, we use the staggered-grid approach with a gauge transformation $[28,29,32]$,

$$
\bar{\psi}=\psi e^{i \chi}, \quad \bar{A}=A+\frac{\vec{\chi}-\chi}{h_{x}}, \quad \bar{B}=B+\frac{\chi^{\uparrow}-\chi}{h_{y}},
$$

where $h_{x}$ and $h_{y}$ are the mesh size in the $x$ - and $y$-directions, and the arrows are used to represent the neighbouring points in the appropriate direction. The discretized average magnetization $M$ (in units of $H_{0}$ ) is then given by

$$
M=\frac{\sum_{p}\left(\frac{\vec{B}-B}{h_{x}}-\frac{A^{\uparrow}-A}{h_{y}}\right)}{p}-H,
$$

where $p$ is the whole number of grid points in a sample. $A^{\uparrow}$ and $\vec{B}$ indicate the neighbouring location on grid of $A$ in the $x$-direction and $B$ in the $y$-direction, respectively [32].

The effect of transport current can be included into the simulation system by different ways [22-25]. In the present work, we introduce the applied external current $I$ (with corresponding density $J$ in units of $j_{0}$ ) along the $x$-direction via the electric potential $\phi=J x$ in eq. (1) and neglect the contribution of the constant term $\sigma \nabla \phi$ in eq. (2) for simplicity [25]. In addition, we set the metalsuperconductor boundary condition (with $b=20>0$ ) for the sample outer edges perpendicular to the $x$-direction, but $1 / b=0$ for the other edges. When a system is subjected to a constant dc current and a time-periodic ac current along the $x$-direction, we consider sinusoidal ac plus static dc currents [26], and the total current density is given by

$$
J=J_{d c}+\left|J_{a c}\right| \sin (2 \pi t / T)
$$

where $T$ is the period of the current oscillations. Note that the external ac signal is spatially uniform as the dc current biasing. 
In this work, we take the intervals of cell points $h_{x} / \lambda=$ $h_{y} / \lambda=0.15$ and $\Delta t / \tau=0.05$. We choose the computational grid $32 h_{x} \times 32 h_{y}$ for all square samples, which is optimal to obtain reliable results in minimal time. The samples are first cooled below the critical temperature with the Meissner state as the initial state, and then a transport current parallel to the $x$-axis is applied. Note that we neglect the thermal fluctuation term in order to reveal mainly the influences of the applied current and magnetic field on the V-Av dynamics. Meanwhile, our approach without taking into account the demagnetization effects could be applied to a very thin film (with thickness $d$ ) when the lateral dimensions of the sample are much smaller than the Pearl length $\Lambda=2 \lambda^{2} / d[19]$.

The sample with a centered hole. - We begin our analysis by comparing the dynamics of (anti)vortices in a symmetric mesoscopic superconducting system with a small centered hole in the presence of an applied ac current or a constant dc current. As representative examples, fig. 2 shows several snapshots of the contour plots of the magnetic-field distribution at zero applied external magnetic field for a square loop with a centered $3 h_{x} \times 3 h_{y}$ hole. The red and blue region denotes the current-induced vortex and antivortex, respectively. The upper panels (a)-(c) correspond to the case of a dc driven current with $J_{d c}=$ 0.007, and the bottom panels (d)-(f) correspond to an ac current case with amplitude $\left|J_{a c}\right|=0.008$ and period $T=2000$. We choose the GL parameter $\kappa=6$. To exhibit clearly the (anti)vortex motion inside the sample, we will only plot the magnetic-field profile of the superconducting region in the following.

In figs. 2(a) and (d) we can clearly see that a $\mathrm{V}-\mathrm{Av}$ pair is created perpendicular to the direction of the applied current (the supercurrent exceeds locally the GL depairing current for the given parameters). The supercurrent rotates with opposite direction for the vortex and antivortex, respectively. For the dc current case, a V-Av pair nucleates at the opposite inner boundaries of the sample and moves in opposite directions under the influence of the Lorentz force toward the outer boundaries of the sample (fig. 2(b)). After the expulsion, a new pair is created again in the middle of the sample, continuing the evolution process (fig. 2(c)). By contrast, differently from the dc current flowing only in one direction, the ac current periodically reverses direction along the $x$-axis. When an ac current is applied, a V-Av pair can also nucleate at the inner edges of the sample and moves toward the outer edges (fig. 2(d)), which is similar to the dc current case. However, the velocities of both the vortex and the antivortex decrease due to the reduced magnitude of the driven current in one halfperiod of the ac signal. Thus, a stable $\mathrm{V}-\mathrm{Av}$ pair forms temporarily. After that, with increasing magnitude of the ac current in another half-cycle, a new $\mathrm{Av}-\mathrm{V}$ pair will be created near the hole boundaries. In the meanwhile, the existed V-Av pair moves backward because of the reversed Lorentz force (see fig. 2(e)). Consequently, the vortex
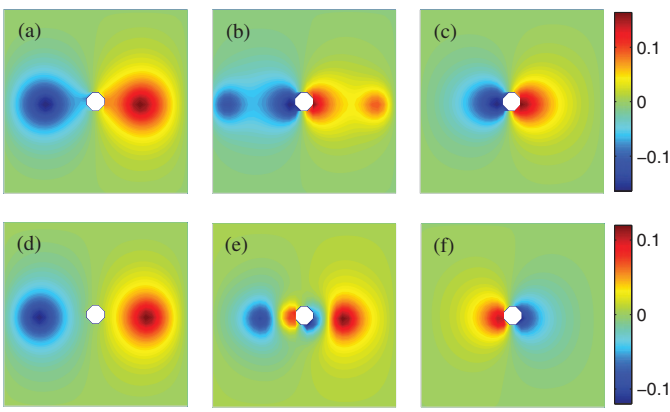

Fig. 2: (Colour on-line) Snapshots of the magnetic-field profile for a mesoscopic superconducting loop with a centered hole size $3 h_{x} \times 3 h_{y}$ at zero external magnetic field. The upper and bottom panels correspond to the case of a dc driven current with $J_{d c}=0.007$ and an ac current case with amplitude $\left|J_{a c}\right|=$ 0.008 and period $T=2000$, respectively.

and the antivortex collide together on their route in both branches of the sample (see fig. 2(f)). Notice that the $\mathrm{V}$-Av collision process is sensitive to the amplitude and the period of the ac current. A decreased/increased current amplitude leads to a weaker/stronger ac forcing, and then the speed of (anti)vortices becomes slower/faster in the vortex channel. Moreover, for a decreased current period the $\mathrm{V}-\mathrm{Av}$ pair cannot propagate deeper inside the sample because of the increased frequency of the current oscillation. On the contrary, further increasing the period of the ac drive results in expulsion of the vortex and antivortex from the outer edges simultaneously in each half-period of the current oscillation. Thus, no V-Av collision process can occur in both cases.

Next we would like to demonstrate the effect of the external magnetic field $H$ normal to the $x$ - $y$ plane on the dynamics of $\mathrm{V}-\mathrm{Av}$ pairs in the sample subjected to a timeperiodic ac current. We only consider the weak-field case in that no flux can penetrate and enter the sample at the outer edge. Note that when a magnetic field is applied, the system generates a Meissner current which compensates the injected current in one branch of the sample while it adds to the one in the other branch of the sample generating a preferential condition for the nucleation of (anti)vortices [22]. Figure 3 shows the time evolution of magnetization $M$ for the sample under different external magnetic field $H$ in the presence of an ac current with $\left|J_{a c}\right|=0.008$ and $T=2000$. Clearly, with increasing $H$ the oscillatory curves show a zigzag-like behavior with a local minimum of $M$ in one period. Namely, the $\mathrm{V}-\mathrm{Av}$ collision and annihilation process can occur. The inserts (1)-(5) show the snapshots of the magnetic-field profile of the sample at time points indicated in the black solid curve for $H=0.15$. At the times near point 1 , where the magnitude of the ac drive is almost zero, only the flow of a single vortex is observed. This vortex moves slowly and then stops for a while inside the sample (see the inset (1)). Later on, it moves toward the hole under a reversed applied drive, and at the same time a new $\mathrm{V}-\mathrm{Av}$ 


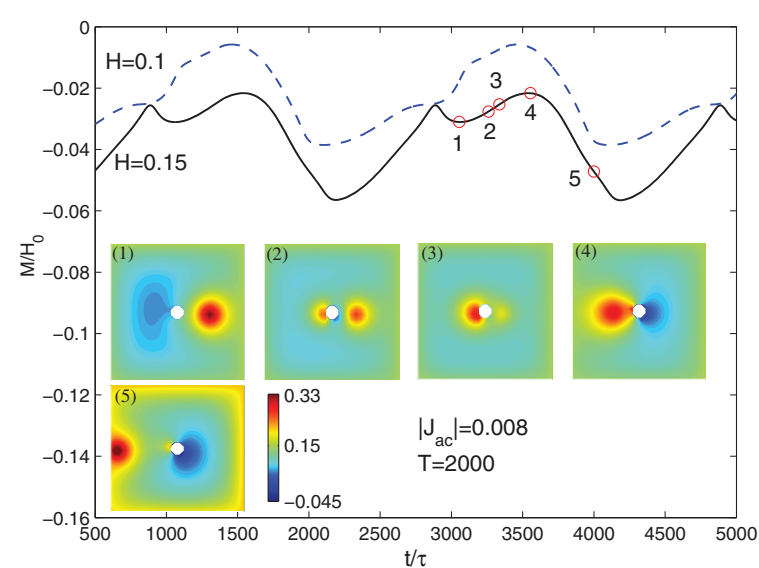

Fig. 3: (Colour on-line) Magnetization $M$ as a function of time $t$ for a mesoscopic superconducting loop with a centered $3 h_{x} \times 3 h_{y}$ hole when $\left|J_{a c}\right|=0.008$ and $T=2000$ at $H=0.1$ (blue dashed curve) and $H=0.15$ (black solid curve). The inset panels show the contour plots of the magnetic-field distribution at different time points depicted on the solid curve.

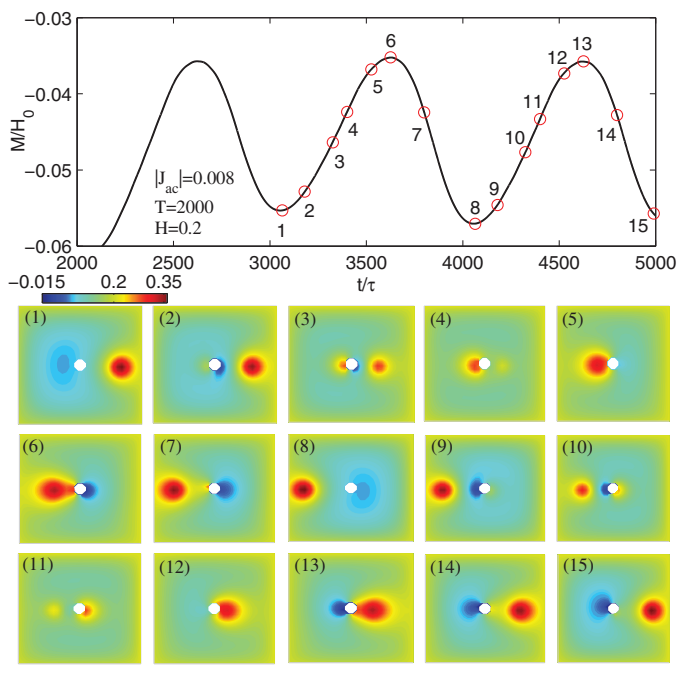

Fig. 4: (Colour on-line) Same as in fig. 3 but now for $H=0.2$.

pair tends to nucleate near the hole edges as well (see the inset (2)). As the single vortex moves forward to the hole, at some moment it will collide with the antivortex created from the hole and then annihilate each other (see the inset (3)). During the collision process, another vortex nucleates near the centered hole and enter the other branch of the sample (see the inset (4)). Under the applied drive, it keeps moving toward the outer surface and finally leaves the sample (see the inset (5)).

More interestingly, a further increased applied field can result in considerable modulation of the magnetization curve: a periodic sinusoidal-like oscillation is observed and the frequency is twice the ones of the ac signal and the voltage, as shown in fig. 4 for $H=0.2$. To clearly characterize the evolution behavior of the $\mathrm{V}-\mathrm{Av}$ dynamics, the bottom panels (1)-(15) display the contour plots of the

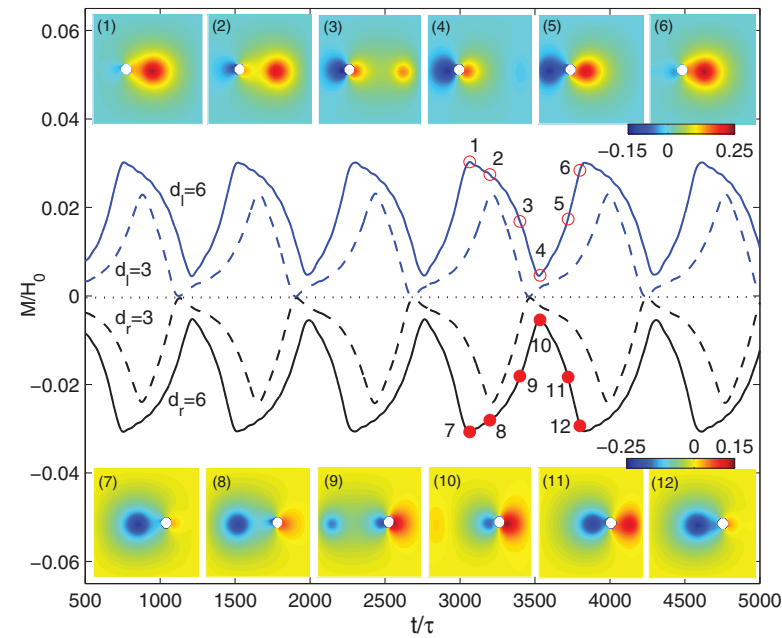

Fig. 5: (Colour on-line) $M$ - $t$ curve in a mesoscopic superconducting loop with a decentered $3 h_{x} \times 3 h_{y}$ hole for different left distances $d_{l}$ (blue curves) and right ones $d_{r}$ (black curves) from the sample center when $J_{d c}=0.007$ and $H=0$.

magnetic-field distribution at times indicated in the curve in one-period of the ac current. In the time interval from point 1 (where the ac signal reverses its direction) to 6 , we can observe the same dynamical evolution of the $\mathrm{V}-\mathrm{Av}$ collision and annihilation as in fig. 3 reflected in the increase in the average magnetization. After the point 7, a single vortex enters the sample and moves toward the left outer edge under applied drive (see the panel (7)). However, vortex velocity is inversely proportional to the applied magnetic field. As a consequence of the further enlarged field, the single vortex slows down and stops inside the sample. Thus, a steady vortex state will be temporally formed again when the ac signal reverses direction (see the panel (8)). That is to say, we arrive again at the initial state similar to the point 1 , while the following dynamical process is opposite to the one shown above (see the panels (9)-(15)).

The sample with an off-centered hole. - In this section, we perform to describe the case of asymmetric mesoscopic superconducting loops with an off-centered $3 h_{x} \times 3 h_{y}$ hole and examine the influence of the offcentered distance of the hole on the V-Av dynamics under a current drive in the absence or presence of an external magnetic field.

We first study the dynamics of (anti)vortices in asymmetric loops when the system is subjected to a dc current drive. Notice that, due to the off-centered hole, the $\mathrm{V}$-Av pair will not nucleate in the middle of the sample. Namely, the creation point (i.e., the hole) of $\mathrm{V}-\mathrm{Av}$ pairs shift away from the center of the sample. As a result, a single vortex will appear longer or shorter than the corresponding antivortex due to the sample asymmetry. Figure 5 depicts the time evolution of the average magnetization when $J_{d c}=0.007$ and $H=0$, where $d_{l}$ 


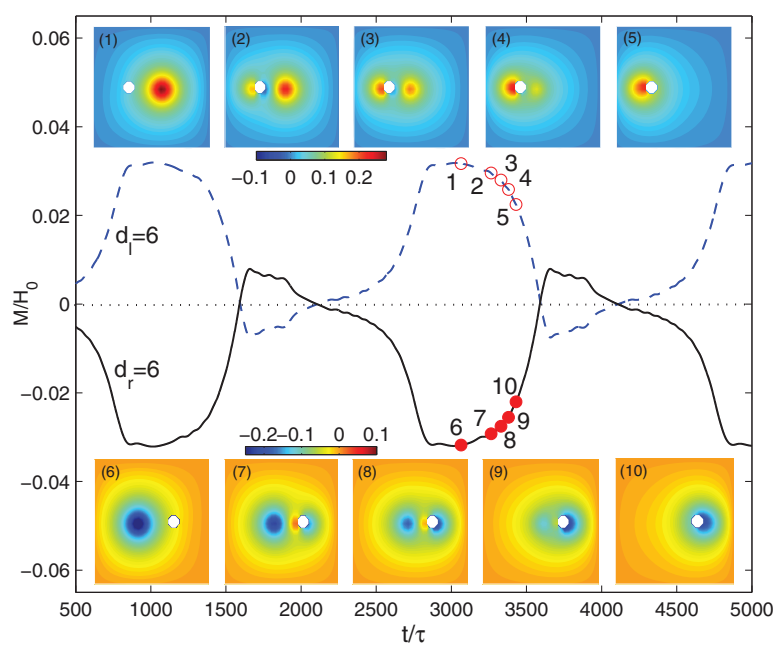

Fig. 6: (Colour on-line) $M-t$ curve in a mesoscopic superconducting loop with a decentered $3 h_{x} \times 3 h_{y}$ hole for $d_{l}=6$ (the dashed curve) and $d_{r}=6$ (the solid curve) in the presence of an applied ac current with $\left|J_{a c}\right|=0.008$ and $T=2000$ when $H=0$.

and $d_{r}$ denote the displacement of the hole from the center along the vortex channel as shown in fig. 1. We notice that for a sample with a left/right off-centered hole, an absolute paramagnetic-like/diamagnetic-like response can be realized in the zero-external-magnetic-field regime. As an example, the insets (1)-(6) and (7)-(12) in fig. 5 show the snapshots of the magnetic-field distribution for the case of $d_{l}=6$ (blue solid curve) and $d_{r}=6$ (black solid curve) in one period, respectively. We find that the (anti)vortices exit the sample one by one due to different distances reaching the external border of the sample. Meanwhile, with increasing $d_{l}$ or $d_{r}$ the lift time of $\mathrm{V}-\mathrm{Av}$ pairs becomes smaller (compare time scales in the solid and dashed curves).

For comparison, we next consider the asymmetric sample under a time-periodic (and spatially uniform) ac drive. As representative examples, we investigate two samples with off-centered distances $d_{l}=6$ and $d_{r}=6$ under an applied ac current with the amplitude $\left|J_{a c}\right|=0.008$ and period $T=2000$. Figure 6 gives the time evolution of magnetization in the absence of an external magnetic field, together with snapshots of the magnetic-field profile of the sample. For the sample with a left off-centered hole (see the blue dashed curve and the insets (1)-(5)), we find that the process of $\mathrm{V}-\mathrm{Av}$ collision and annihilation can only take place in the wider branch of the sample in contrast to the symmetric case in figs. 2(d)-(f). This evolution behavior is very similar to the case of a symmetric sample in the presence of an applied magnetic field in fig. 3. Noticeably, this event manifests itself as a paramagnetic-like response in the magnetization. Moreover, for the sample with a right off-centered hole, one can expect an opposite evolution of dynamical process from the black solid curve in fig. 6. Because of the asymmetric geometry only

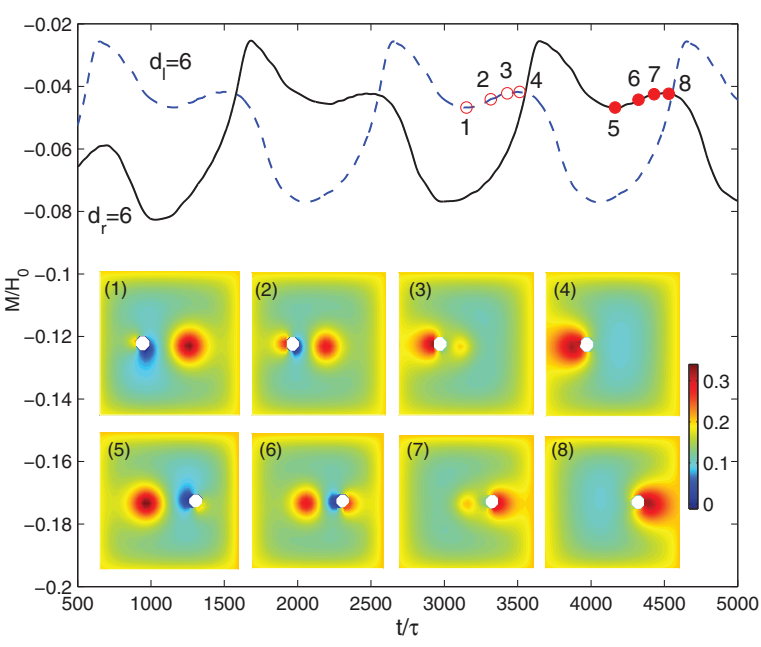

Fig. 7: (Colour on-line) Same as in fig. 6 but now for $H=0.2$.

the flow of an antivortex is observed when the ac signal tends to reverse its direction (see the inset (6)). With increasing reversed current drive, this antivortex moves oppositely and will encounter a vortex generated near the hole (see the insets (7)-(10)). Namely, the $A v-V$ collision process occurs inside the sample corresponding to a diamagnetic-like response in the $M-t$ curve.

Note that the $A v-V$ collision process can only show up in the case of zero external magnetic field. Figure 7 demonstrates the influence of the applied magnetic field on the dynamical evolution for an asymmetric sample with same parameters used as in fig. 6. For the cases of different off-centered distances with $d_{l}=6$ (dashed curve) and $d_{r}=6$ (solid curve), we cannot find the sinusoidal-like oscillation shown in fig. 4 for a symmetric sample. Especially, only the $\mathrm{V}-\mathrm{Av}$ collision and annihilation process can be observed in the right branch of the sample for the case of $d_{l}=6$ (see the insets $\left.(1)-(4)\right)$ and in the left branch for the case of $d_{r}=6$ (see the insets (5)-(8)), respectively.

Conclusions. - In summary, using the TDGL formalism, we mainly studied the transport properties of the mesoscopic square loop with a small hole in the presence of a time-periodic ac current. At zero applied magnetic field the vortex and the antivortex nucleate in pairs at the inner edges of the sample and move in opposite directions toward the outer surface under the influence of Lorentz force. Differently from the case of a constant dc current, the existing V-Av pair moves backward when the ac signal reverses its direction and then collides with a new $\mathrm{Av}-\mathrm{V}$ pair created near the centered hole. When a weak magnetic field is applied, this $\mathrm{V}-\mathrm{Av}$ annihilation process can only be observed in one branch of the sample due to the effect of magnetic field on the (anti)vortex velocity. Interestingly, a sinusoidal-like oscillatory modulation of the magnetization curve is observed for a further enlarged field, and the periodic dynamical process of the VAv annihilation occurs in both branches of the sample. We further investigated the $\mathrm{V}-\mathrm{Av}$ dynamics in the asymmetric 
sample with an off-centered hole. In this case, the creation point of V-Av pairs shifts away from the center of the sample. The nucleation and annihilation process of $\mathrm{V}-\mathrm{Av}$ pairs turns out to be very different from the symmetric case. An absolute paramagnetic-like/diamagnetic-like response can be realized for a sample with a left/right off-centered hole in the zero-external-magnetic-field regime, and the $A v-V$ collision process cannot be found in the system under an ac signal when an external magnetic field is applied.

With the development of experimental techniques, it is now possible to fabricate microstructured samples with different shapes and to introduce nanoscale structures (e.g. holes) into a superconductor. The reported dynamic phenomena in our study reveal a method for manipulating (anti)vortex velocity and the lifetime of V-Av pairs by applied magnetic field and current in mesoscopic superconductors. Moreover, one can experimentally consider breaking of the sample symmetry according to our results, in order to fine tune the process of the $\mathrm{V}-\mathrm{Av}$ collision and annihilation. We expect that our theoretical results will be useful for futuristic applications in superconducting electronics.

We are grateful to GoliBJon BerdiYOROv for useful discussions. This work was supported by NSF China under Grant Nos. 61371020 and 61271163, by Visiting Scholar Program of Shanghai Municipal Education Commission, by Innovation Program of Shanghai Municipal Education Commission under Grant No. 13YZ006, and by Flemish Science Foundation (FWO-Vl).

\section{REFERENCES}

[1] Filby E. T., Zhukov A. A., de Groot P. A. J., Ghanem M. A., Bartlett P. N. and Metlushko V. V., Appl. Phys. Lett., 89 (2006) 092503.

[2] Veldhorst M., Molenaar C. G., Wang X. L., Hilgenkamp H. and Brinkman A., Appl. Phys. Lett., 100 (2012) 072602.

[3] Moshchalkov V. V., Gielen L., Strunk C., Jonckheere R., QIU X., van Haesendonck C. and Bruynseraede Y., Nature (London), 373 (1995) 319.

[4] Chibotaru L. F., Ceulemans A., Bruyndoncx V. and Moshchalkov V. V., Nature (London), 408 (2000) 833.

[5] Geim A. K., Dubonos S. V., Lok J. G. S., Henini M. and MaAn J. C., Nature (London), 396 (1998) 144.

[6] Geim A. K., Dubonos S. V., Grigorieva I. V., Novoselov K. S., Peeters F. M. and Schweigert V. A., Nature (London), 407 (2000) 55.

[7] Chibotaru L. F., Ceulemans A., Bruyndoncx V. and Moshchalkov V. V., Phys. Rev. Lett., 86 (2001) 1323.
[8] Misko V. R., Fomin V. M., Devereese J. T. and Moshchalkov V. V., Phys. Rev. Lett., 90 (2003) 147003.

[9] Geurts R., Milosevic M. V. and Peeters F. M., Phys. Rev. Lett., 97 (2006) 137002.

[10] Geurts R., Milosevic M. V. and Peeters F. M., Phys. Rev. B, 79 (2009) 174508.

[11] Van Bael M. J., Bekaert J., Temst K., Van Look L., Moshchalkov V. V., Bruynseraede Y., Howells G. D., Grigorenko A. N., Bending S. J. and Borghs G., Phys. Rev. Lett., 86 (2001) 155.

[12] Lange M., Bael M. J. V., Bruynseraede Y. and Moshchalkov V. V., Phys. Rev. Lett., 90 (2003) 197006.

[13] Lange M., Van Bael M. J., Silhanek A. V. and Moshchalkov V. V., Phys. Rev. B, 72 (2005) 052507.

[14] Milosevic M. V. and Peeters F. M., Phys. Rev. Lett., 93 (2004) 267006.

[15] Milosevic M. V. and Peeters F. M., Phys. Rev. Lett., 94 (2005) 227001.

[16] Milosevic M. V., Berdiyorov G. R. and Peeters F. M., Phys. Rev. B, 75 (2007) 052502.

[17] Geurts R., Milosevic M. V., Albino Aguiar J. and Peeters F. M., Phys. Rev. B, 87 (2013) 024501.

[18] Wen Lin, Zha Guo-Qiao and Zhou Shi-Ping, EPL, 102 (2013) 27004.

[19] Sardella E., Malvezzi A. L., Lisboa-Filho P. N. and Ortiz W. A., Phys. Rev. B, 80 (2009) 012506.

[20] Lima Clessio L. S. and De Souza Silva Clecio C., Phys. Rev. B, 80 (2009) 054514.

[21] Kapra A. V., Misko V. R., Vodolazov D. Y. and Peeters F. M., Supercond. Sci. Technol., 24 (2011) 024014 .

[22] Berdiyorov G. R., Milosevic M. V. and Peeters F. M., Phys. Rev. B, 79 (2009) 184506.

[23] Berdiyorov G. R., Milosevic M. V. and Peeters F. M., Physica C, 470 (2010) 946.

[24] Berdiyorov G. R., Hernandez-Nieves A. D., Milosevic M. V., Peeters F. M. and Dominguez D., Phys. Rev. B, 85 (2012) 092502.

[25] ChaO X. H., Zhu B. Y., Silhanek A. V. and Moshchalkov V. V., Phys. Rev. B, 80 (2009) 054506.

[26] Berdiyorov G. R., Savelev S. E., Milosevic M. V., Kusmartsev F. V. and Peeters F. M., Phys. Rev. B, 87 (2013) 184510.

[27] Berdiyorov G. R., Savelev S. E., Kusmartsev F. V. and Peeters F. M., Supercond. Sci. Technol., 26 (2013) 125010.

[28] Coskun E. and Kwong M. K., Nonlinearity, 10 (1997) 579.

[29] Kim S., Hu C.-R. and Andrews M. J., Phys. Rev. B, 69 (2004) 094521.

[30] DE Gennes P. G., Superconductivity of Metals and Alloys (Addison-Wesley, New York) 1994.

[31] Zha Guo-Qiao, Zhou Shi-Ping, Zhu Bao-He and Shi Yao-Ming, Phys. Rev. B, 73 (2006) 104508.

[32] Kaper H. G. and Kwong M. K., J. Comput. Phys. B, 119 (1995) 120. 